\title{
SOFT GAMMA-RAY REPEATERS IN NEARBY GALAXIES: RATE, LUMINOSITY FUNCTION, AND FRACTION AMONG SHORT GAMMA-RAY BURSTS
}

\author{
Eran O. Ofek ${ }^{1}$ \\ Received 2006 August 3; accepted 2006 November 22
}

\begin{abstract}
It was suggested that some of the short-duration gamma-ray bursts (GRBs) are giant flares of soft gamma-ray repeaters (SGRs) in nearby galaxies. To test this hypothesis, I have constructed a sample of 47 short GRBs, detected by the Interplanetary Network (IPN), for which the position is constrained by at least one annulus on the celestial sphere. For each burst, I have checked whether its IPN $3 \sigma$ error region coincides with the apparent disk of one of 316 bright, star-forming galaxies found within $20 \mathrm{Mpc}$. I find a single match of GRB 000420B with M74, which could, however, be due to a chance coincidence. I estimate the IPN efficiency as a function of fluence and derive the galaxy sample completeness. I find that assuming there is a cutoff in the observed energy distribution of SGR flares at $\leq 10^{47} \mathrm{ergs}$, the fraction of SGRs among short GRBs with fluence above $\sim 10^{-5} \mathrm{ergs} \mathrm{cm}^{-2}$ is $<16 \%(95 \%$ confidence). I estimate the number of active SGRs in each one of the galaxies in the sample, and combine it with the distances to these galaxies, the IPN efficiency, and the SGR flare energy distribution, to derive the rate of giant flares with energy above $4 \times 10^{46}$ ergs. I find that the rate of such giant flares is about $(0.4-5) \times 10^{-4} \mathrm{yr}^{-1}$ per SGR. This rate is marginally consistent with the observed Galactic rate. Comparison of the Galactic rate with the inferred extragalactic rate implies a gradual cutoff (or steepening) of the flare energy distribution at $\leqslant 3 \times 10^{46} \mathrm{ergs}(95 \%$ confidence). Using the Galactic SGR flare rate, I set a lower limit of $1 \%$ on the fraction of SGR flares among short GRBs.
\end{abstract}

Subject headings: galaxies: individual (M74, NGC 7331) — gamma rays: bursts — stars: neutron

Online material: machine-readable table

\section{INTRODUCTION}

The rate of soft gamma-ray repeater (SGR) giant flares and their fraction among short-duration gamma-ray bursts (GRBs; Kouveliotou et al. 1993) are important ingredients for the understanding of giant flares in the context of the magnetar model (Duncan \& Thompson 1992; Paczyński 1992; for a recent review see Woods \& Thompson 2006).

The large energy release from the 2004 December 27 giant flare, combined with some similarities between the temporal and spectral properties of giant flares and short GRBs, has reignited the idea that some or all of the short GRBs are in fact SGR giant flares in nearby galaxies (e.g., Dar 2005; Hurley et al. 2005; Palmer et al. 2005; Nakar et al. 2006). Additional support to this hypothesis came from the observed Galactic rate of giant flares with energy above $4 \times 10^{46} \mathrm{ergs}$, of one in $30 \mathrm{yr}$ among four active Galactic SGRs.

A limit on the fraction of SGRs among short GRBs was obtained by Nakar et al. (2006), who searched the error quadrilateral of six, well-localized, Interplanetary Network (IPN) GRBs for relatively nearby galaxies. They put a upper limit of $<40 \%$ ( $95 \%$ confidence limit [CL]) on the fraction of SGRs among bright short GRBs.

Lazzati et al. (2005), inspected the spectra of 76 Burst And Transient Source Experiment (BATSE) short bursts. They found three GRBs whose spectra are well described by a blackbody model, and 15 bursts for which a fit with a blackbody model is not excluded. Lazzati et al. (2005) then argued, based on the gammaray light curves of these short GRBs, that none of them were likely SGR bursts. They derived a limit on the fraction of SGR bursts among bright BATSE events of $<4 \%(95 \% \mathrm{CL})$. I note that the Lazzati et al. (2005) results are based on the assumption that all

\footnotetext{
${ }^{1}$ Division of Physics, Mathematics and Astronomy, California Institute of Technology, Pasadena, CA.
}

the giant flares have similar light curves. However, this assumption is based on a small number of observed giant flares. If I relax the Lazzati et al. (2005) assumption about the temporal characteristics of giant flares, but keep their assumption regarding the spectral properties of SGRs (and there are up to 18 SGR flares in their sample), their result poses an upper limit on the fraction of SGRs among bright short GRBs of $<35 \%$, at the $95 \%$ CL.

Recently, two possible detections of extragalactic SGRs were claimed: Crider (2006) detected a $13.8 \mathrm{~s}$ periodicity in the tail of the gamma-ray light curve of GRB 970110 . He suggested that this burst may be an SGR flare in NGC 6946. Golenetskii et al. (2005) reported the detection of GRB 051103, which includes the nearby galaxy M81 in its error quadrilateral (see Ofek et al. [2006] and Frederiks et al. [2006] for follow-up observations and discussion).

Another quantity related to the fraction of SGRs among short GRBs is the rate of SGR giant flares. This rate is the consequence of the total energy available for SGRs, which in the context of the magnetar model is provided by the magnetic field of a neutron star.

Recently, Stella et al. (2005) argued that the rate of SGR giant flares, with energy above $5 \times 10^{46} \mathrm{ergs}$, is about $10^{-2} \mathrm{yr}^{-1}$. They obtained this result by using a uniform prior (see, however, Duncan 2001) on the observed rate. They further argued that about 70 giant flares, with energy above $5 \times 10^{46}$ ergs, are expected during the lifetime of an SGR, and concluded that magnetic fields of $B \gtrsim$ $10^{16} \mathrm{G}$ are needed in order to explain the energy source of SGRs.

Popov \& Stern (2006) attempted to find extragalactic SGRs by searching the BATSE catalog for short GRBs that spatially coincide with the Virgo Cluster. They used this search to put an upper limit on the rate of giant flares with energy above $\sim 10^{46}$ ergs, of $10^{-3} \mathrm{yr}^{-1}$. However, this limit was obtained by assuming that $T_{50}$ of all SGR giant flares is in the range of $0.05-0.7 \mathrm{~s}$, and by ignoring the BATSE positional uncertainty. A similar limit was obtained by Palmer et al. (2005). 


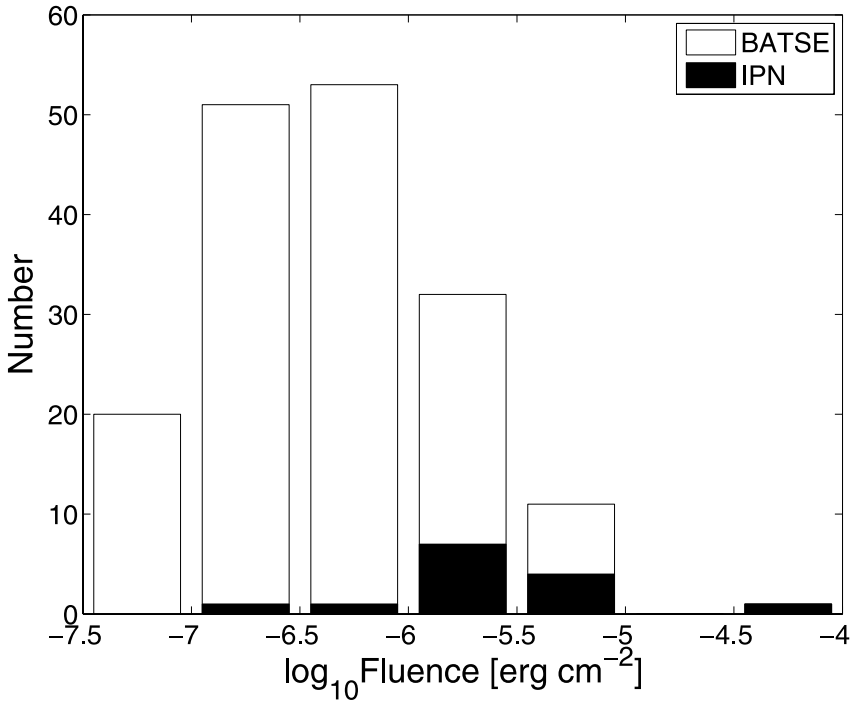

FIG. 1.-Histogram of BATSE GRBs integrated four-channel fluence, for short-duration bursts triggered by BATSE (open bars), and BATSE-triggered short-duration GRBs that were detected by at least two IPN detectors and thus constrained by at least one annulus ( filled bars).

In this paper I match the IPN $(\S 2)$ short GRBs with nearby galaxies $(\S 3)$. Contrary to previous efforts, I do not introduce any assumptions about the temporal or spectral properties of SGR giant flares. I present, in $\S 4$, the search for IPN short-duration GRBs that spatially coincide with nearby galaxies. In $\S 5$ I use the results of this search to calculate the rate of SGR giant flares and confront it with the rate estimated based on the Galactic SGRs. I finally discuss the results in $\S 6$.

\section{THE IPN SENSITIVITY}

The IPN (e.g., Laros et al. 1997; Hurley et al. 1999) is a set of gamma-ray detectors on board several spacecraft in the solar system. The IPN uses GRB photon arrival times to triangulate their celestial positions. Each pair of spacecraft constrain the GRB position to an annulus on the celestial sphere. The area of IPN $3 \sigma$ error regions is typically two orders of magnitude smaller than BATSE error circles. In fact, IPN positions were used to calibrate BATSE positional errors (Briggs et al. 1999).

Here I have use the IPN catalog ${ }^{2}$ containing all IPN-triangulated GRBs observed from 1990 November 12 to 2005 October $31 .^{3}$ Figure 1 shows a histogram of the integrated four-channel fluence for BATSE-triggered short-duration GRBs $\left(T_{90}<2 \mathrm{~s}\right.$; open bars), as well as all short-duration GRBs triggered both by BATSE and additional IPN detector (i.e., that have at least one annulus constraint; filled bars).

Figure 2 shows the approximate all-sky IPN completeness as a function of BATSE integrated four-channel fluence. Completeness here is calculated from the ratio of the two histograms in Figure 1, multiplied by the exposure completeness of the Konus-Wind and BATSE short-duration GRB sample that is presented in the next section. I estimated the exposure completeness to be about $86 \%(=[4 \times 0.48+11 \times 1.0] / 15)$. This is based on the fact KonusWind started to work only in 1994 (11 out of $15 \mathrm{yr}$ ), and the exposure completeness of BATSE, which worked $4 \mathrm{yr}$ without

\footnotetext{
2 See http://www.ssl.berkeley.edu/ipn3/interpla.html, version 2005 December 17. For reference, this IPN catalog version is available from http://astro .caltech.edu/ eran/GRB/IPN/NearbyGal/CatIPN.txt.ver17122005.

3 This catalog does not contain GRB 051103, which may have originated in the nearby galaxy M81 (Ofek et al. 2006; Frederiks et al. 2006).
}

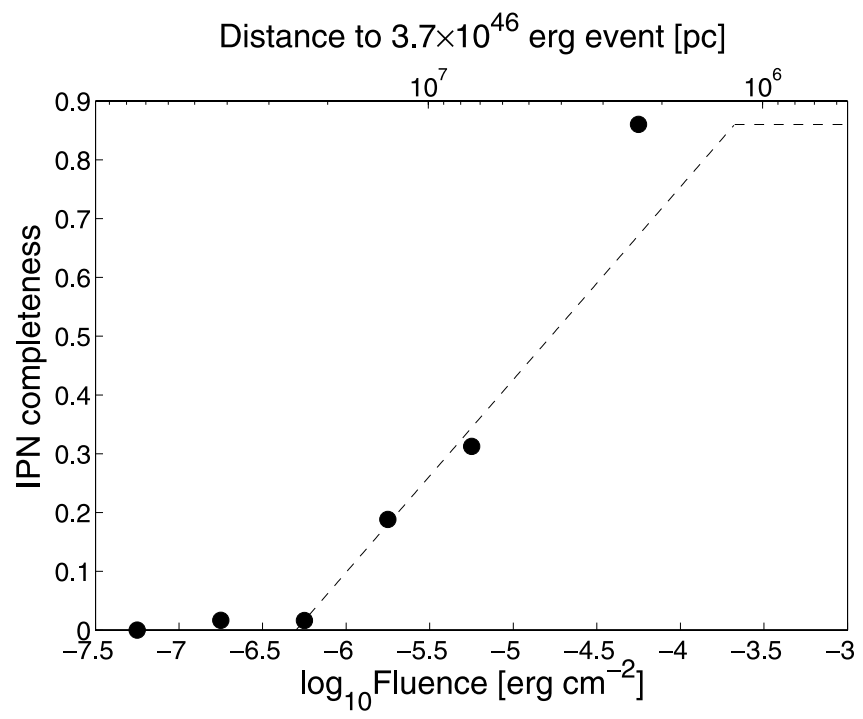

FIG. 2.-All-sky IPN approximate completeness as calculated from the ratio of the two histograms in Fig. 1, multiplied by the exposure completeness ( $86 \%$; see text). The top $x$-axis shows the corresponding distance to a $3.7 \times 10^{46} \mathrm{ergs}$ flare, which is the energy of the 2004 December 27 flare assuming a distance of $15 \mathrm{kpc}$ to SGR 1806-20 (Hurley et al. 2005). The dashed line is an approximation to this completeness function (eq. [1]).

Konus-Wind, is about $48 \%{ }^{4}$ The dashed line shows an approximation to this completeness function given by

$C(F)= \begin{cases}0, & F \leq 5 \times 10^{-7}, \\ 0.328 \log _{10} F+2.065, & 5 \times 10^{-7}<F<2.1 \times 10^{-4}, \\ 0.86, & F \geq 2.1 \times 10^{-4},\end{cases}$

where $F$ is the fluence. BATSE sensitivity is almost complete down to a fluence of about $10^{-6} \mathrm{ergs}^{-2}$, in which the IPN efficiency drops to about $10 \%$. Therefore, the sensitivity function (eq. [1]) is an approximation to the IPN absolute mean efficiency. However, I will show in $\S 3$ that the results are not very sensitive to the exact shape of this completeness function.

\section{SELECTION OF THE GRB AND GALAXY SAMPLES}

I selected from the IPN catalog all GRBs with a $3 \sigma$ error region constrained by at least one annulus with semiwidth smaller than $1^{\circ}(1260)$. I further selected from this list all GRBs that were triggered by BATSE (752) and have $T_{90}<2.0 \mathrm{~s}$ (29 events). The $T_{90}$ duration at which a GRB has an equal probability of being a short- or long-duration GRB is about $T_{90}=5 \mathrm{~s}$ (Donaghy et al. 2006). As SGR giant flares have durations shorter than $2 \mathrm{~s}$, the upper limit on the fraction of SGR giant flares among shortduration GRBs derived in this paper is conservative.

In addition, I searched for short-duration GRBs among the Konus-Wind satellite observations (Cline et al. 2003). A catalog of short GRBs detected by Konus-Wind was published by Mazets et al. (2004); however, some of the GRBs in this catalog were classified as long GRBs by BATSE. Therefore, instead of using this catalog I used the following statistical approach: I inspected by eye the Konus-Wind light curves for which an IPN annulus constraint is available, and selected all the bursts that have a total duration of less than $2 \mathrm{~s}$. Due to the differences between the

\footnotetext{
${ }^{4}$ Calculated from the BATSE all-sky exposure map, available at http://cossc .gsfc.nasa.gov/docs/cgro/cossc/batse/4Bcatalog/4b_exposure.html.
} 


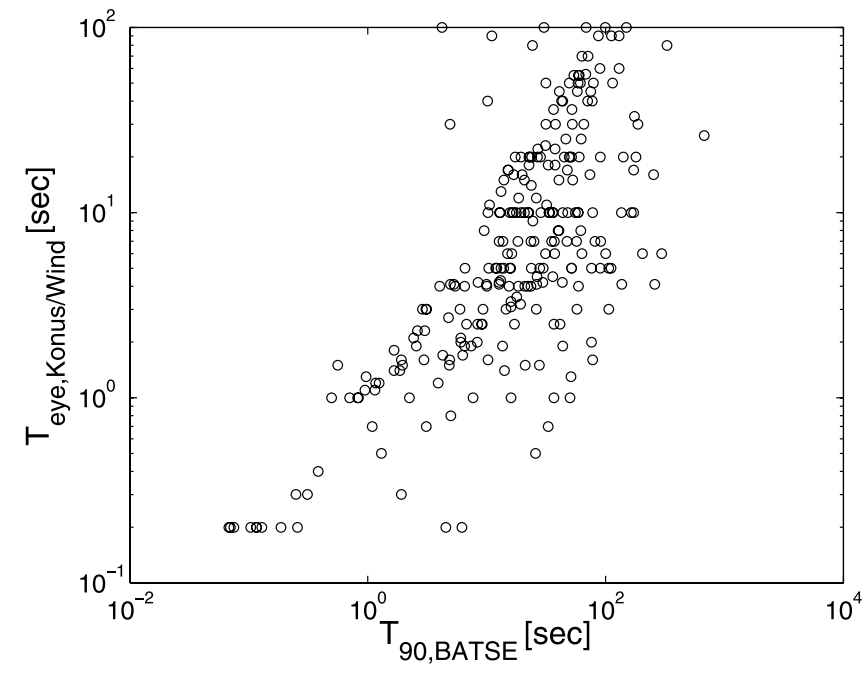

Fig. 3.-BATSE $T_{90}$ vs. the estimated duration (by eye from Konus-Wind light curve), $T_{\text {eye }}$, of the GRBs.

BATSE and Konus-Wind detectors, this sample is contaminated by long GRBs (which appear like short GRBs for the KonusWind detector). Figure 3 shows the BATSE $T_{90}$ versus the duration estimated by eye ( $\left.T_{\text {eye }}\right)$ of GRBs that were triggered by both BATSE and Konus-Wind.

I found 83 Konus-Wind bursts with $T_{\text {eye }} \leq 2 \mathrm{~s}$ and annulus semiwidth of less than $1^{\circ}$. Of these 83 events, 37 were detected by BATSE, of which 23 were classified by BATSE as longduration bursts (i.e., $T_{90}>2 \mathrm{~s}$ ). Therefore, the Konus-Wind sample has $\sim 60 \%$ (23/37) contamination by long GRBs. Given this contamination factor, I estimate that the actual number of short bursts, among these 46 remaining events, is $18_{-4}^{+5}$. Finally, I compiled a list of 75 events: 29 BATSE events with $T_{90}<2 \mathrm{~s}$ and 46 Konus-Wind events with $T_{\text {eye }}<2 \mathrm{~s}$. This list contains about 47 $(\sim 18+29)$ short bursts.
Next, I selected a sample of bright star-forming galaxies within $20 \mathrm{Mpc}$ from a modified version of the Tully (1988) nearby galaxies catalog. The selected galaxies have absolute magnitude $M_{B} \leq$ -18 , reside at distances less than $20 \mathrm{Mpc}$, and have morphological types other than E or S0. Introducing the last criterion, I assume that SGRs form predominantly in young stellar populations (see, however, Levan et al. 2006). I excluded from this list the Large Magellanic Cloud (LMC). The nearby galaxy sample contains 316 galaxies, which are listed along with their basic properties in Table 1. Also listed in this table are the far-infrared (far-IR) fluxes and estimated supernova (SN) rates in these galaxies derived in $\S 5$.

\subsection{The Galaxy Sample Completeness}

There are three factors that affect the completeness of this galaxy sample: (1) galaxies behind the Galactic plane; (2) star formation in galaxies not included in the sample; and (3) the fact that the sample is volume limited. I estimate these incompleteness factors below.

To estimate the completeness of the galaxy sample (Table 1) due to Galactic obscuration, I calculated the number of galaxies in this catalog as a function of Galactic latitude, $b$. I then extrapolated the number of galaxies from the unobscured regions (i.e., $b \sim 30^{\circ}$ ) to the obscured regions. Based on this extrapolation, I estimated the completeness of the galaxy sample to be about $\epsilon_{\mathrm{G}}=90 \%$.

Next, I estimated, $\epsilon_{\mathrm{SFR}}$, the fraction of star formation within galaxies with $M_{B} \leq-18$ relative to the total star formation within $20 \mathrm{Mpc}$. I calculated the far-IR flux ${ }^{5}$ of the galaxies, defined by $2.58 f_{60}+f_{100}$, where $f_{60}$ and $f_{100}$ are the 60 and $100 \mu \mathrm{m}$ fluxes, respectively. This quantity is a good estimator of the star formation rate in galaxies (Helou et al. 1988). Given the galaxy distances, I summed the absolute far-IR flux of all the galaxies (excluding

\footnotetext{
${ }^{5}$ Based on Infrared Astronomical Satellite (IRAS) Point-Source Catalog, version 2.0, IPAC (1986).
}

TABLE 1

Nearby Bright Galaxies

\begin{tabular}{|c|c|c|c|c|c|c|c|c|}
\hline Name & $\begin{array}{l}\text { R.A., Decl. } \\
(\mathrm{J} 2000.0)\end{array}$ & $\begin{array}{c}B_{T}^{\mathrm{a}} \\
(\mathrm{mag})\end{array}$ & $\begin{array}{l}M_{B}^{\mathrm{b}, \mathrm{c}} \\
(\mathrm{mag})\end{array}$ & $\begin{array}{l}\text { Angular Diameter } \\
\text { (arcmin) }\end{array}$ & $\begin{array}{c}\text { Distance }^{\mathrm{c}, \mathrm{d}} \\
(\mathrm{Mpc})\end{array}$ & Type $^{\mathrm{e}}$ & $\begin{array}{l}\text { IR Flux }{ }^{\mathrm{f}} \\
\text { (Jy) }\end{array}$ & $\begin{array}{l}\text { SN rate } \\
\left(\mathrm{yr}^{-1}\right)\end{array}$ \\
\hline N7814 ................ & $000318,+160900$ & 10.92 & -20.12 & 5.9 & 16.2 & 2 & 0.000 & 0.00000 \\
\hline $\mathrm{N} 14 \ldots \ldots \ldots \ldots \ldots \ldots$ & $000848,+154900$ & 12.10 & -18.59 & 2.9 & 13.7 & 10 & 0.000 & 0.00000 \\
\hline N55 ..................... & $001454,-391100$ & 7.50 & -18.22 & 34.1 & 1.4 & 9 & 202.119 & 0.00245 \\
\hline N178................... & $003906,-141100$ & 12.77 & -18.70 & 2.1 & 19.7 & 9 & 0.000 & 0.00000 \\
\hline $\mathrm{N} 224 \ldots \ldots \ldots \ldots \ldots \ldots$ & $004242,+411600$ & 3.56 & -20.82 & 193.2 & 0.7 & 3 & 793.507 & 0.00279 \\
\hline N247.................... & $004706,-204500$ & 8.66 & -18.10 & 22.2 & 2.2 & 7 & 8.335 & 0.00026 \\
\hline $\mathrm{N} 253 \ldots \ldots \ldots \ldots \ldots \ldots$ & $004736,-251800$ & 7.34 & -20.20 & 25.8 & 3.2 & 5 & 3100.177 & 0.20000 \\
\hline $\mathrm{N} 278 \ldots \ldots \ldots \ldots \ldots \ldots$ & $005206,+473300$ & 10.75 & -19.76 & 2.7 & 12.6 & 3 & 106.233 & 0.10603 \\
\hline $0102-06 \ldots \ldots \ldots$. & $010506,-061300$ & 11.70 & -19.15 & 4.4 & 14.8 & 7 & 5.152 & 0.00703 \\
\hline N406..................... & $010724,-695300$ & 11.90 & -19.44 & 2.7 & 18.5 & 3 & 7.853 & 0.01685 \\
\hline N628.................... & $013642,+154700$ & 9.61 & -20.47 & 7.0 & 10.4 & 5 & 19.736 & 0.01331 \\
\hline N7331 ................. & $223706,+342600$ & 9.67 & -21.26 & 9.7 & 15.3 & 4 & 131.932 & 0.19339 \\
\hline
\end{tabular}

Notes.-Table 1 is published in its entirety in the electronic edition of the Astrophysical Journal. A portion is shown here for guidance regarding its form and

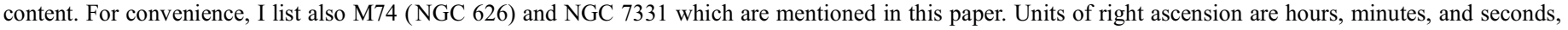
and units of declination are degrees, arcminutes, and arcseconds.

a Apparent blue magnitude, $B_{T}$, corrected for Galactic extinction and for the external galaxy extinction (see Tully 1988).

b Absolute blue magnitude as calculated from the corrected distance and apparent extinction-corrected magnitude.

c The distances listed in the Tully (1988) catalog are based on galaxy recession velocities, assuming an Hubble constant $H_{0}=75 \mathrm{~km} \mathrm{~s}^{-1} \mathrm{Mpc}^{-1}$. I corrected all the distances in this catalog by a factor of 75/70 (i.e., to convert to $H_{0}=70 \mathrm{~km} \mathrm{~s}^{-1} \mathrm{Mpc}^{-1}$ ), and recalculated the absolute magnitude accordingly.

${ }^{\mathrm{d}}$ Distances are based on recession velocities, assuming $H_{0}=70 \mathrm{~km} \mathrm{~s}^{-1} \mathrm{Mpc}^{-1}$.

e Galaxy type, see Tully (1988) for details.

${ }^{\mathrm{f}}$ The total observed far IR flux, $2.58 f_{60}+f_{100}$, calculated by summing the fluxes of the IRAS sources found within the galaxy radius from the galaxy position.

g SN rate estimated by normalizing the absolute far IR flux to that of NGC 253, and assuming this galaxy has $0.2 \mathrm{SN}^{-1}$ (Pietsch et al. 2001). 


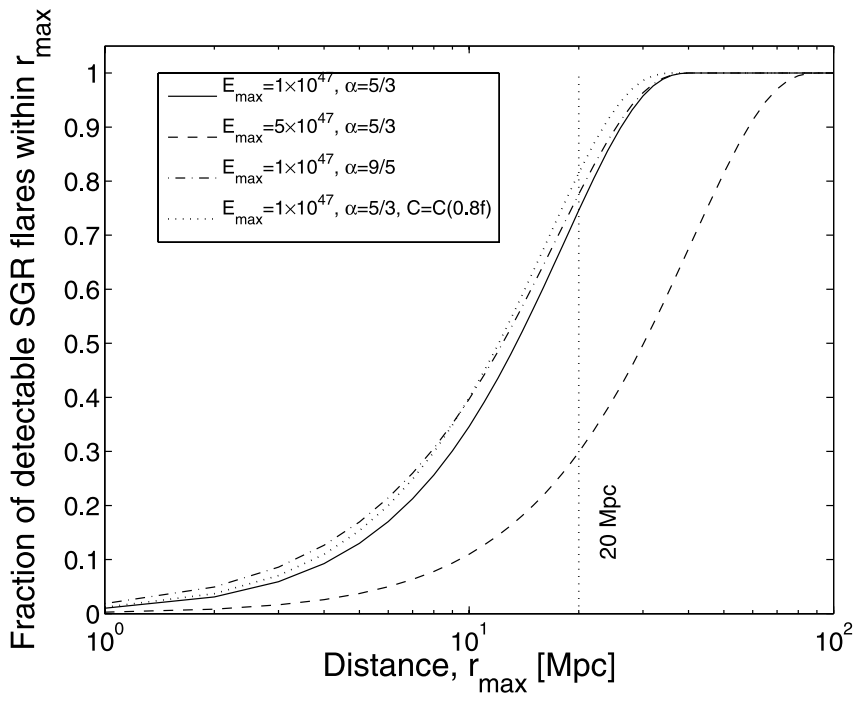

FIG. 4.-Survey volume completeness, $\epsilon_{V}$ (eq. [2]), as a function of distance $r_{\max }$, for four cases: $E_{\max }=10^{47} \mathrm{ergs}, \alpha=5 / 3$ (solid line); $E_{\max }=5 \times 10^{47} \mathrm{ergs}$, $\alpha=5 / 3$ (dashed line); $E_{\max }=10^{47}$ ergs, $\alpha=9 / 5$ (dash-dotted line); and $E_{\max }=$ $10^{47} \mathrm{ergs}, \alpha=5 / 3$, and a modified completeness function [eq. (1); $C=C(0.8 \mathrm{~F})$; dotted line $]$.

E0/S0) brighter than $M_{B}<-18$ within $10 \mathrm{Mpc}$ and divided it by the absolute far-IR flux of all galaxies (excluding E0/S0) brighter than $M_{B}<-12$. Note that I use a distance cut of $10 \mathrm{Mpc}$ in order to avoid incompleteness due to missing faint galaxies. I found that $\epsilon_{\mathrm{SFR}}=95 \%$.

The galaxy sample contains galaxies within $20 \mathrm{Mpc}$. However, SGR giant flares can be observed to larger distances. To estimate the survey volume completeness, I divide the expected number of SGR flares (assuming constant number density of SGRs) that can be observed by the IPN up to a distance of $r_{\max }$, by the total observable number of IPN SGR flares:

$$
\epsilon_{V}=\frac{\int_{0}^{r_{\max }} 4 \pi r^{2} \int_{0}^{E_{\max }} C\left(E / 4 \pi r^{2}\right) E^{-\alpha} d E d r}{\int_{0}^{\infty} 4 \pi r^{2} \int_{0}^{E_{\max }} C\left(E / 4 \pi r^{2}\right) E^{-\alpha} d E d r},
$$

where $r$ is the distance, $C(F)$ is the completeness function (eq. [1]), and $E_{\max }$ is the break in the flare energy distribution. I weighted the number of flares of energy $E, d N / d E$, by the empirical $E^{-\alpha}$ factor, with $\alpha=5 / 3$ (Cheng et al. 1996).

Figure 4 shows $\epsilon_{V}$ as a function of the distance $r_{\max }$, for four different combinations of $E_{\max }, \alpha$ and $C(F)$ (see legend). It is clear that the volume completeness is not sensitive to small changes in $\alpha$, nor to the exact shape of the completeness function. However, the completeness, $\epsilon_{V}\left(r_{\max }=20 \mathrm{Mpc}\right)$ is sensitive to the break energy, $E_{\max }$. Figure 5 shows the volume completeness, $\epsilon_{V}\left(r_{\max }=\right.$ $20 \mathrm{Mpc}$ ), as a function of $E_{\max }$. A reasonable value for $E_{\max }$ is $10^{47} \mathrm{ergs}$, which roughly corresponds to the total magnetic energy available for a $B=10^{15} \mathrm{G}$ neutron star. Assuming $E_{\max }=$ $10^{47} \mathrm{ergs}$, the volume-completeness of our survey is $\epsilon_{V}\left(r_{\max }=\right.$ $20 \mathrm{Mpc})=0.75$, while if $E_{\max }=5 \times 10^{47} \mathrm{ergs}$, then the survey completeness drops to $30 \%$ (see, however, $\S 5$ ).

\section{THE SEARCH FOR SGR FLARES FROM NEARBY GALAXIES}

For each burst in the short GRB sample, I searched for overlap between its IPN $3 \sigma$ error region and the apparent disk of one of the galaxies in Table 1. The IPN constraints include one or more annuli on the celestial sphere. In addition to the annuli, the IPN database occasionally contains constraints from individual instru-

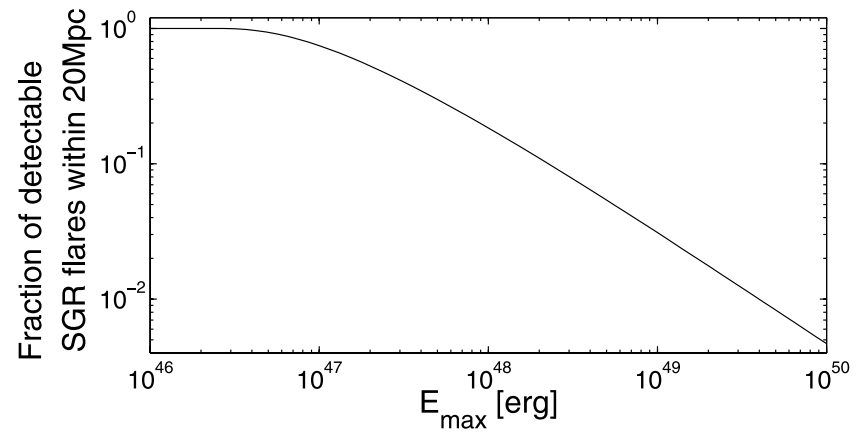

FIG. 5.- Survey volume completeness, $\epsilon_{V}$ (eq. [2]), for $r_{\max }=20 \mathrm{Mpc}$, as a function of the break in the flare energy distribution, $E_{\max }$ (assuming $\alpha=5 / 3$ ).

ments: ecliptic latitude constraints from Konus-Wind; error radii from various satellites; and/or planet blocking regions (i.e., in cases that Earth or Mars blocked part of the detector field of view). Some of these error regions (e.g., BATSE; WATCH [Sazonov et al. 1998], COMPTEL [Kippen et al. 1998], EGRET [Gonzalez et al. 2004], PHEBUS [Tkachenko et al. 2002], SIGMA [Claret et al. 1994], BeppoSAX [Guidorzi et al. 2004], and HETE [Vanderspek et al. 2003]) are given at the $1 \sigma$ confidence. For these error radii I added known systematic errors (i.e., $1.6^{\circ}$ for BATSE; Briggs et al. 1999) and translated all the errors, which I assumed are circular ${ }^{6}$ and normally distributed, to $3 \sigma$ errors.

To avoid incompleteness due to the positional inaccuracies of the Tully (1988) catalog, the galaxy diameter used in the search was increased by $1^{\prime}$.

I have found two matches between the short GRB candidates and the galaxies listed in Table 1. The first, GRB 000420B, coincides with the position of the nearby Sc-type galaxy M74. Figure 6 shows a Galaxy Evolution Explorer (GALEX) near UV-band image, emphasizing the star-forming regions in M74, with the IPN annulus overlayed (solid lines). GRB 000420B was detected by Konus-Wind at UTC 2000 April $2011: 44: 31,{ }^{7}$ and its gammaray light curve is shown in Figure 7.

Note that I did not detect, using a Scargle (1982) periodogram, any periodic signal in the light curve within $\sim 100 \mathrm{~s}$ after the GRB spike.

The second match, GRB 000526B coincides with the position of the galaxy NGC 7331. However, as seen in Figure 8, this galaxy has an elongated shape (while in the search I assumed the galaxies are round) and the IPN annulus does not coincide with the galaxy apparent disk or any star-forming regions within this galaxy.

I note there is a smaller galaxy within the NGC 7331 group that does fall within the IPN annulus. However, this galaxy does not pass the absolute magnitude cut, and the a priori probability of finding an SGR flare from such a galaxy is small. Therefore, I conclude that GRB 000526B is unlikely to be associated with NGC 7331.

I have estimated the probability for a chance coincidence between the short-GRB IPN error regions and the galaxy sample. This was done by randomizing the positions of the 316 galaxies over the celestial sphere and searching for a match with one of the IPN short-GRBs in the list. I repeated this simulation 1000 times and found that the expectancy number of matches is $\sim 3$ and the probability of finding two matches is $\sim 15 \%$, and three or more matches is $\sim 76 \%$. Note that since the actual galaxy positions are

\footnotetext{
${ }^{6}$ Note that error regions for GRB positions obtained by some of the spacecraft are not circular.

${ }^{7}$ On April 20, M74 is about $6^{\circ}$ from the Sun.
} 


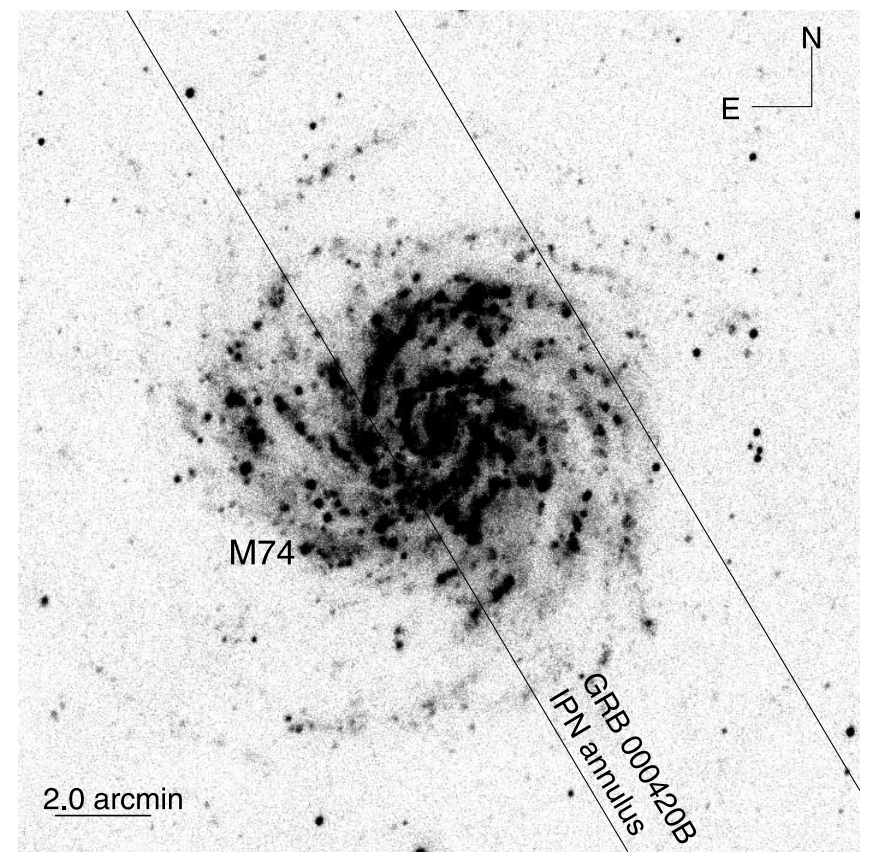

FIG. 6.-GALEX near-UV-band image of the Sc-type galaxy M74 (NGC 628). The parallel lines mark the section of the IPN annulus of GRB 000420B that passes through the galaxy. The IPN constraints for this burst include an annulus whose parameters are the following: annulus center at $\alpha=303.76^{\circ}, \delta=-29.4^{\circ}$ (J2000.0); annulus radius of $89.63^{\circ}$ and semiwidth of $0.04^{\circ}$; and a Konus-Wind ecliptic latitude constraint that indicates that the GRB ecliptic latitude is between $+2^{\circ}$ to $+22^{\circ}\left(3 \sigma\right.$; the ecliptic latitude of M74 is $\left.+5.2^{\circ}\right)$. The total area of this annulus segment is $3.25 \mathrm{deg}^{2}$.

correlated and the IPN constraints are coordinate-dependent (e.g., Konus-Wind ecliptic latitude constraint), this simulation does not mimic the search process accurately. However, it indicates that such a chance coincidence is possible. Therefore, I cannot securely identify GRB 000420B with M74.

Given the product of the completeness factors, $\epsilon_{\mathrm{G}} \epsilon_{\mathrm{SFR}} \epsilon_{V} \approx 64 \%$ (see $\S 3$ ), and conservatively assuming that the search yielded a single SGR candidate among 47 short GRBs, I put an upper limit on the fraction of SGRs among short GRBs of $<16 \%(<27 \%)$, at the one-sided 95\% (99.73\%) CL (Gehrels 1986). Assuming that GRB 000420B is indeed an SGR flare in M74, the fraction of SGRs among short GRBs is about $3 \%\left[=1 /\left(47 \epsilon_{\mathrm{SFR}} \epsilon_{\mathrm{G}} \epsilon_{V}\right)\right]$.

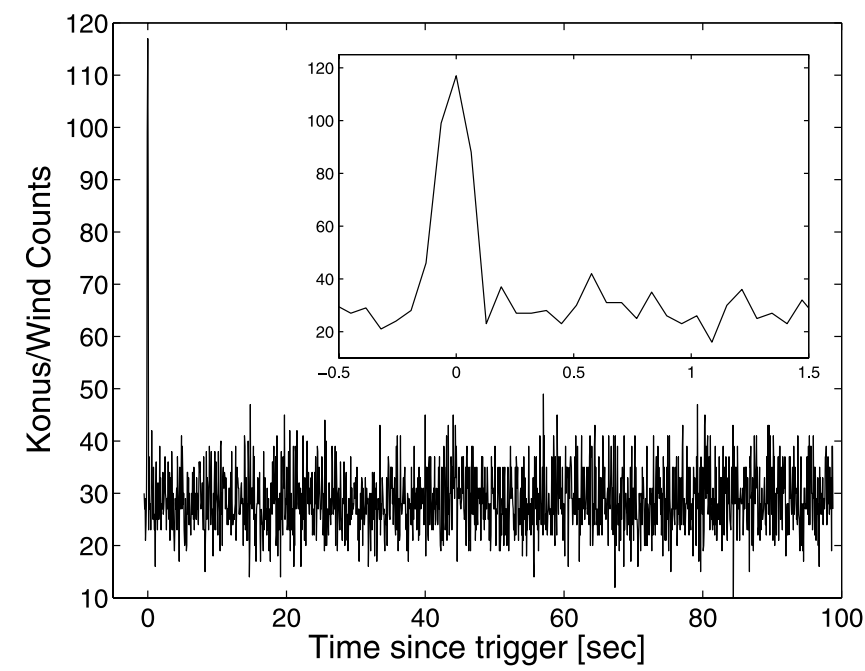

FIG. 7.-Konus-Wind gamma-ray light curve of GRB 000420B, which may have originated in M74. The GRB shows a single, short ( $0.3 \mathrm{~s}$ width), peak.

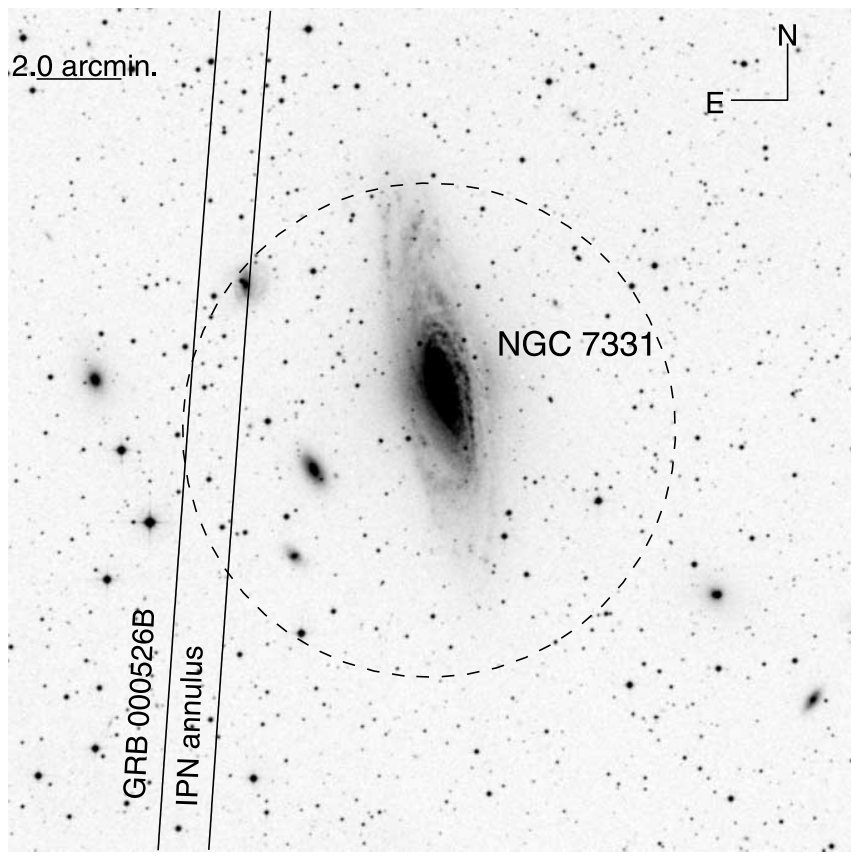

FIG. 8.- R-band image of the galaxy NGC 7331 from the Palomar Observatory Sky Survey II. The parallel solid lines mark the section of the IPN annulus of GRB 000526B that passes near this galaxy. The center of the dashed-line circle around NGC 7331 shows the actual position of NGC 7331 in the Tully (1988) catalog, which is accurate only to about $1^{\prime}$. To avoid any incompleteness caused by the positional inaccuracies, I increased the galaxy radii by $1^{\prime}$ compared to the radii listed in Tully (1988). The circle size corresponds to the increased galaxy radius.

\section{THE RATE OF GIANT FLARES}

A closely related quantity to the fraction of SGR giant flares among short GRBs is the rate of SGR giant flares, which I estimate below.

Among the four active SGRs in the Galaxy (including the LMC), a single giant flare with energy above $3.7 \times 10^{46}$ ergs was observed in the last $30 \mathrm{yr}$. Therefore, the derived rate of giant flares with energy above $3.7 \times 10^{46} r_{15}^{2}$ ergs (Hurley et al. 2005), is $\left(120_{-100}^{+5100}\right)^{-1} \mathrm{yr}^{-1}$ per SGR (95\% confidence interval; CI; Gehrels 1986), where $r_{15}$ is the distance to SGR $1806-20$ in $15 \mathrm{kpc}$ units (Corbel \& Eikenberry 2004).

Next, I estimate based on the results of $\S 4$, the rate of SGR giant flares, and compare it with the rate derived from the Galactic SGR giant flare. Assuming that all the SGRs have identical flare properties (i.e., the same cutoff energy $E_{\max }$ and $\alpha$ ), then the rate of giant flares, with energy above $E_{\mathrm{gf}}$, per year per SGR, $f_{\mathrm{gf}}$, is given by

$$
f_{\mathrm{gf}}\left(\geq E_{\mathrm{gf}}, E_{\mathrm{max}}\right)=\frac{\sum_{i} N_{o, i}}{\Delta T} \frac{\int_{E_{\mathrm{gf}}}^{E_{\max }} E^{-\alpha} d E}{\sum_{i} N_{i}^{\mathrm{SGR}} \int_{0}^{E_{\max }} E^{-\alpha} C\left(E / 4 \pi r_{i}^{2}\right) d E},
$$

where $\Delta T$ is the time span of observations (15 yr), $N_{o, i}$ is the observed number of SGR flares in the $i$ th galaxy $\left(\sum_{i} N_{o, i} \leq 1\right)$, $\alpha=5 / 3$ (Cheng et al. 1996), $N_{i}^{\text {SGR }}$ is the number of active SGRs in the $i$ th galaxy, and $C$ is the completeness function (eq. [1]). To estimate $N_{i}^{\text {SGR }}$, I use the total far-IR flux (see $\S 3$ ) of the galaxies (Table 1) as an estimator for the star formation rate (Helou et al. 1988). I converted the far-IR flux to the $\mathrm{SN}$ rate, $R_{i}^{\mathrm{SN}}$, by normalizing the far-IR flux of each galaxy to that of NGC 253, which I assumed has $0.2 \mathrm{SN} \mathrm{yr}^{-1}$ (Pietsch et al. 2001). The far-IR flux along with the derived SN rate for each galaxy in this sample are 


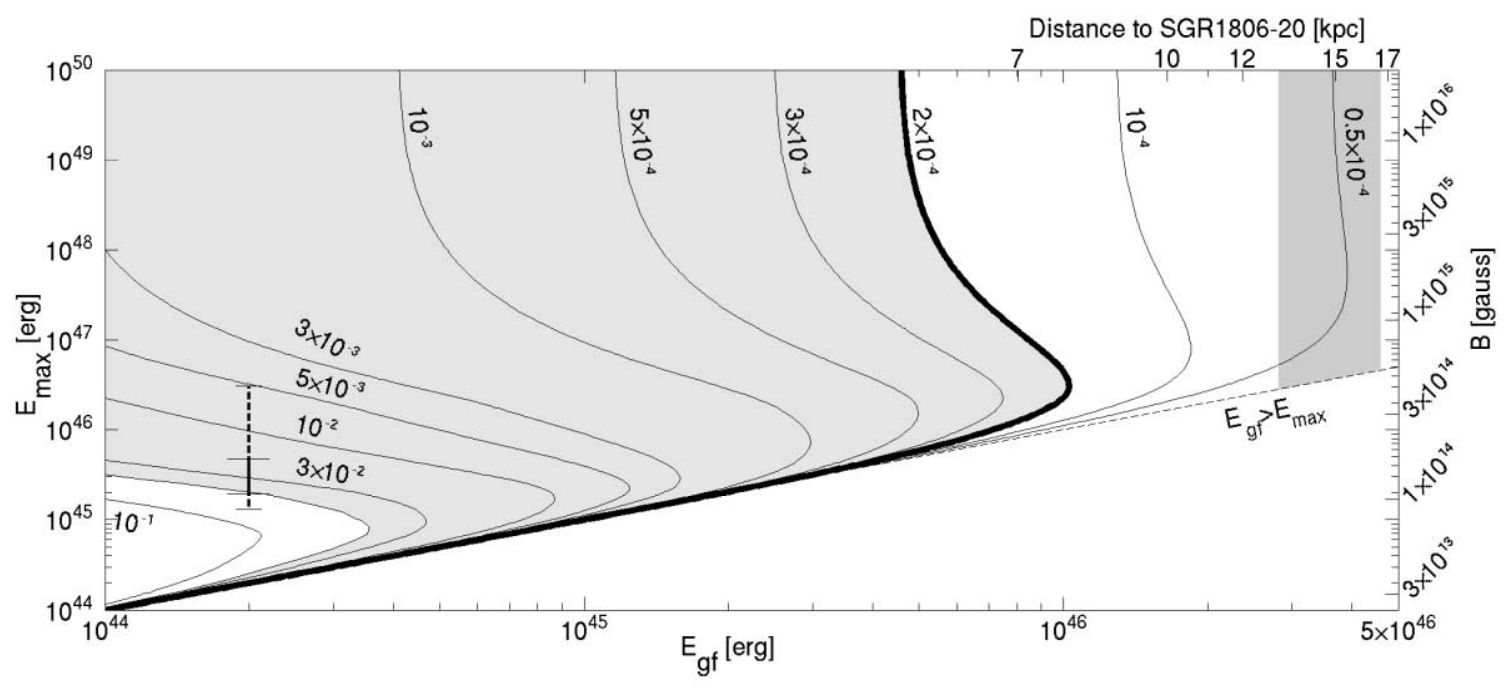

Fig. 9.-Contours of equal $f_{\mathrm{gf}} / \sum_{i} N_{o, i}$, as a function of the giant flare energy, $E_{\mathrm{gf}}$, and the cutoff energy, $E_{\mathrm{max}}$. The labels on the contours are the SGR giant flares rate in $\mathrm{yr}^{-1}$ (i.e., rate of events for which $E>E_{\mathrm{gf}}$ ). The triangular area in the bottom right is the unphysical region in which $E_{\mathrm{gf}}>E_{\max }$. The $95 \%$ confidence region of giant flare rate (per SGR), with $E>3.7 \times 10^{46} \mathrm{ergs}$, as implied by the Galactic SGR observations, (i.e., $0.0083_{-0.0081}^{+0.039} \mathrm{yr}^{-1}$ ), is shown as the light-gray region on the left side of the figure, and the $2 \sigma$ lower limit (i.e., approximately $\left.2 \times 10^{-4} \mathrm{yr}^{-1}\right)$ is marked by a heavy line. The estimated energy of the 2004 December $27 \mathrm{giant}$ flare, $(3.7 \pm$ $0.9) \times 10^{46}$ ergs assuming a distance of $15 \mathrm{kpc}$ to SGR $1806-20$, is marked as a dark-gray region on the right side of the figure. The error bar on the left-hand side marks the rate of Galactic SGR giant flares, with $E>2 \times 10^{44} \mathrm{ergs}$. The $1 \sigma \mathrm{CI}$ is marked by a solid line and the $2 \sigma \mathrm{CI}$ is marked by a dashed line. For convenience, I plot on the top axis of the figure the distance to SGR 1806-20, which corresponds to the energy $E_{\mathrm{gf}}$, of the 2004 December 27 giant flare. On the right axis I plot the approximate magnetic field, $B$, corresponding to $E_{\max }\left[=\left(B^{2} / 8 \pi\right)(4 / 3) \pi R_{n s}^{3}\right]$, where I set the neutron star radius, $R_{\mathrm{ns}}$, to $10 \mathrm{~km}$.

listed in Table 1. Finally, the number of SGRs in the $i$ th galaxy, $N_{i}^{\mathrm{SGR}}$, is

$$
N_{i}^{\mathrm{SGR}} \cong N_{\mathrm{MW}}^{\mathrm{SGR}} \frac{R_{i}^{\mathrm{SN}}}{R_{\mathrm{MW}}^{\mathrm{SN}}}
$$

where, $N_{\mathrm{MW}}^{\mathrm{SGR}}$ is the number of active SGRs in the Milky-Way galaxy (four), and the $\mathrm{SN}$ rate in our Galaxy, $R_{\mathrm{MW}}^{\mathrm{SN}}$, was set to $0.03 \mathrm{yr}^{-1}$.

Now I can compare the observed Galactic rate of giant flares with the rate implied by the extragalactic survey. Figure 9 shows contours of equal $f_{\mathrm{gf}} / \sum_{i} N_{o, i}$ (i.e., the rate $f_{\mathrm{gf}}$ in eq. [3] calculated assuming a single SGR flare was detected in the sample, $\left.\sum_{i} N_{o, i}=1\right)$, as a function of the giant flare energy $E_{\mathrm{gf}}$ and the cutoff energy, $E_{\max }$. The labels on the contours denote the SGR giant flare rate in $\mathrm{yr}^{-1}$ per SGR. The $95 \%$ confidence region on the rate of giant flares, with energy above $3.7 \times 10^{46} \mathrm{ergs}$, as implied by the Galactic SGR observation (i.e., $0.0083_{-0.0081}^{+0.039} \mathrm{yr}^{-1}$ per SGR) is shown as the light-gray region on the left side of the figure, and the $2 \sigma$ lower limit (i.e., approximately $2 \times 10^{-4} \mathrm{yr}^{-1}$ ) is marked as a bold line. The estimated energy with its uncertainty, of the 2004 December 27 giant flare, assuming a distance of $15 \mathrm{kpc}$ to SGR 1806-20, is marked as dark-gray region on the right side of the figure. For convenience, I plot on the top axis of Figure 9 the distance to SGR 1806-20, which corresponds to the energy of the 2004 December 27 giant flare. Plotted on the rightaxis is the approximate magnetic field, $B$, corresponding to $E_{\max }$ $\left[=\left(B^{2} / 8 \pi\right)(4 / 3) \pi R_{\mathrm{ns}}^{3}\right.$, where I set the neutron star radius, $R_{\mathrm{ns}}$, to $10 \mathrm{~km}]$.

Apparently, there is a conflict between the Galactic rate of SGR giant flares and the rate implied by the extragalactic survey, which predicts a giant flare rate of $\sim 0.5 \times 10^{-4} \mathrm{yr}^{-1}$ (assuming $\left.E_{\mathrm{gf}} \sim 3.7 \times 10^{46} \mathrm{ergs}\right)$. There are several ways to explain this contradiction: the observed number of SGR flares in the search, $\sum_{i} N_{o, i}=1$, is subject to Poisson error $1_{-0.98}^{+4.7}(95 \% \mathrm{CL})$; the estimate of $N_{i}^{\mathrm{SGR}}$ (eq. [4]) is subject to errors through the SN rate estimate, and to Poisson error in the number of active SGRs in the Galaxy $\left(4_{-3}^{+6} ; 95 \% \mathrm{CL}\right)$; or the distance to SGR 1806-20 may be smaller than $15 \mathrm{kpc}$ (e.g., $12 \mathrm{kpc}$ [Figer et al. 2004], or 6-10 kpc [Cameron et al. 2005]) and therefore shift the darkgray zone (i.e., energy of the 2004 December 27 giant flare) in Figure 9 to the left. Moreover, the value of the power-law index $\alpha$ may be different from 5/3 (e.g., Götz et al. 2006; $\alpha=1.9$ ), and the luminosity function model is an approximation. In reality the energy distribution (i.e., $d N / d E \propto E^{-5 / 3}$ ) is probably not terminated abruptly at $E_{\max }$, as different SGRs may be born with different magnetic fields. I note that although the tentative detection of a single extragalactic SGR has a large Poisson uncertainty $\left(1_{-0.98}^{+4.6}\right.$, at the $\left.95 \% \mathrm{CL}\right)$, the actual rate cannot be much smaller than one, or it will not be consistent with the observed Galactic rate of giant flares with energy above $3.7 \times 10^{46}$ ergs.

Interestingly, the Galactic rate of giant flares with energy above $2 \times 10^{44}$ ergs is $\left(40_{-20,-26}^{+47,+154}\right)^{-1} \mathrm{yr}^{-1}$ per SGR (The errors are the 1 and $2 \sigma \mathrm{CL}$, respectively; assuming that three ${ }^{8}$ giant flares were observed in $30 \mathrm{yr}$ ). This rate is indicated by the error bar in the left-hand side of Figure 9. The solid line is the $1 \sigma \mathrm{CI}$, and the dashed line is the $2 \sigma \mathrm{CI}$ implied by the Galactic rate. In order to reconcile the Galactic rate with the extragalactic rate, a gradual break (or steepening) in the flare energy distribution is required at $\lesssim 3 \times 10^{46}$ ergs (at the $95 \% \mathrm{CL}$ ).

I note that if I adopt the value of $\alpha=1.9$ found by Götz et al. (2006), then the inferred rate of giant flares with energy above $4 \times 10^{46}$ ergs will be even lower (by about $10 \%$ ) than the rate based on $\alpha=5 / 3$. Moreover, for $\alpha=1.9$ I find that $E_{\max } \lesssim 3 \times 10^{47}$, at the $95 \% \mathrm{CL}$.

To conclude, this analysis shows that $E_{\max } \lesssim 3 \times 10^{46}$ ergs at the $95 \% \mathrm{CL}$; the rate of SGR giant flares is $(0.4-5) \times 10^{-4} \mathrm{yr}^{-1}$ per SGR; and the fraction of SGR flares among short-duration

8 These are 1979 March 5 (SGR 0526-66),1998 August 27 (SGR 1900+14), and 2004 December 27 (SGR 1806-20). Note that SGR flares are correlated (see $\S 6$ ), and therefore Poisson statistics can be used only if the flares have originated from different objects-as in this case. 
GRBs cannot be much smaller than $1 \%$, otherwise it will be inconsistent with the Galactic rate.

\section{DISCUSSION}

The fraction of SGR flares among short GRBs and the rate of SGR giant flares are closely related issues. Measurements of these quantities are important for the understanding of the SGR flare mechanism and magnetic field strength. Below I discuss the possibility of observing additional extragalactic flares and the implications of the measured SGR giant flare rate for the magnetic field strength in the context of the magnetar model.

The derived fraction of SGRs among short GRBs implies that about five to $\sim 65$ extragalactic SGR flares were observed by BATSE. Moreover, I estimate that the Swift satellite may find an extragalactic SGR every several years, and that the Gamma Ray Large Area Space Telescope will localize about 0.2-3 extragalactic SGRs per year. Based on the analysis presented in this paper, it would be surprising if both GRB 000420B and the recently discovered GRB 051103 (Golenetskii et al. 2005; Ofek et al. 2006; Frederiks et al. 2006) were not associated with M74 and M81, respectively.

Verifying that a GRB is indeed an extragalactic SGR flare requires either the detection of persistent $\mathrm{X}$-ray emission, which is beyond our current observational capabilities, or the detection of additional flare activity. The fact that SGR flares are correlated may increase the probability of observing additional flare activity from extragalactic SGRs. The observed time between lowenergy SGR flares follows a lognormal distribution (Laros et al. 1987; Hurley et al. 1994; Cheng et al. 1996). Therefore, it is more appropriate to express the typical time between bursts using the lognormal mean, $M$, and standard deviation, $S$. Assuming a flare rate of $2 \times 10^{-4} \mathrm{yr}^{-1}$, then $\tau$, the expectation value of the time between giant flares, with energy above $3.7 \times 10^{46} \mathrm{ergs}$, is $5000 \mathrm{yr}$. Since the expectancy value of lognormal distribution is given by $\exp \left(M+S^{2} / 2\right)$, and assuming $S=3.46$ (Hurley et al. 1994) is independent of energy, the $\log$ mean for giant flares is $M_{\mathrm{gf}} \cong$ 2.53. Interestingly, the cumulative lognormal distribution suggests that given these parameters (i.e., $M_{\mathrm{gf}}=2.53 ; S=3.46$ ), the probability to observe a second giant flare of an SGR within $10 \mathrm{yr}$ of the first flare (given a sufficient energy source) is $47 \%$. Therefore, if GRB 000420B is associated with M74, and/or GRB 051103 is associated with M81, there is a fair chance to see another flare from these galaxies in the next decade. Furthermore, I note that if more than one burst per SGR will be observed, the use of Poisson statistics will considerably overestimate the flare rate.

As discussed in $\S 1$, Stella et al. (2005) argued that about 70 giant flares, with energy above $5 \times 10^{46} \mathrm{ergs}$, are expected from an SGR during its lifetime, and claimed that SGR magnetic fields are in excess of $10^{16} \mathrm{G}$. My analysis, however, shows that the giant flare rate is 2 orders of magnitude smaller than that estimated by Stella et al. (2005), and therefore, magnetic fields of $B \approx 10^{15} \mathrm{G}$ can provide the required energy source. The giant flare rate I derive, $(0.4-5) \times 10^{-4} \mathrm{yr}^{-1}$ per SGR, suggests that within the lifetime of an SGR, $10^{3}-10^{5} \mathrm{yr}$, it would have of order unity giant flares with energy above $4 \times 10^{46}$ ergs.

To summarize, I searched for a spatial coincidence of IPN GRBs with galaxies within $20 \mathrm{Mpc}$. I have found a single SGR candidate, possibly located within M74. However, this match could be a chance coincidence. Currently, the best way to test the hypothesis that GRB 000420B arose in M74 is to detect additional SGR flares from this galaxy. As SGR activity is correlated, the expected waiting time to a second flare from the same SGR is not necessarily long. I use the results to place an upper limit on the fraction of SGRs among short GRBs with fluence above $\sim 10^{-5} \mathrm{ergs} \mathrm{cm}^{-2}$, of $<16 \%$ at the $95 \% \mathrm{CL}$. This limit is consistent with the recent finding of Nakar et al. (2006) and Lazzati et al. (2005), but contrary to the latter paper it does not depend on the assumption that all SGR giant flares have the same light curve and spectrum. I note that this limit is based on the assumption that magnetars form predominantly in young stellar populations (see, however, Levan et al. 2006). I roughly estimate the efficiency of the IPN to detect giant flares in each of the galaxies in the sample, and find that the rate of giant flares, with energy above $3.7 \times 10^{46} \mathrm{ergs}$, is $(0.4-5) \times 10^{-4} \mathrm{yr}^{-1}$ per SGR. Finally, I show that the SGR flare luminosity function is consistent with a single power-law with a gradual cutoff or steepening below $3 \times 10^{46} \mathrm{ergs}\left(3 \times 10^{47} \mathrm{erg}\right)$, at the $95 \% \mathrm{CL}$, for $\alpha=5 / 3(1.9)$.

I am grateful to Kevin Hurley for his assistance in my use of the IPN data and for maintaining this important database, and to an anonymous referee for useful comments. I thank Shri Kulkarni, Ehud Nakar, Re'em Sari, Orly Gnat, Avishay Gal-Yam, and Brad Cenko, for valuable discussions. This work is supported in part by grants from NSF and NASA.

\section{REFERENCES}

Briggs, M. S., Pendleton, G. N., Kippen, R. M., Brainerd, J. J., Hurley, K., Connaughton, V., \& Meegan, C. A. 1999, ApJS, 122, 503

Cameron, P. B., et al. 2005, Nature, 434, 1112

Cheng, B., Epstein, R. I., Guyer, R. A., \& Young, C. 1996, Nature, 382, 518

Claret, A., et al. 1994, A\&A, 287, 824

Cline, T. L., et al. 2003, in AIP Conf. Proc. 662, Gamma-Ray Burst and Afterglow Astronomy 2001: A Workshop Celebrating the First Year of the HETE Mission, ed. G. R. Ricker \& R. K. Vanderspek (Melville: AIP), 143 Corbel, S., \& Eikenberry, S. S. 2004, A\&A, 419, 191

Crider, A. 2006, in AIP Conf. Proc. 836, Gamma-Ray Bursts in the Swift Era, ed. S. S. Holt, N. Gehrels, \& J. A. Nousek (Melville: AIP), 64

Dar, A. 2005, GCN 2942, http://gcn.gsfc.nasa.gov/gen/gen3/2942.gcn3

Donaghy, T. Q., et al. 2006, ApJ, submitted (astro-ph/0605570)

Duncan, R. C. 2001, in AIP Conf. Proc. 586, 20th Texas Symposium on Relativistic Astrophysics, ed. J. C. Wheeler \& H. Martel. (Melville: AIP), 495

Duncan, R. C., \& Thompson, C. 1992, ApJ, 392, L9

Figer, D. F., Najarro, F., \& Kudritzki, R. P. 2004, ApJ, 610, L109

Frederiks, D. D., Pal'shin, V. D., Aptekar', R. L., Golenetskii, S. V., Cline, T. L.,

\& Mazets, E. P. 2006, Astron. Lett, 33, 19

Gehrels, N. 1986, ApJ, 303, 336
Golenetskii, S., et al. 2005, GCN Circ., 4197, http://gcn.gsfc.nasa.gov/gcn/gcn3/ 4197.gcn3

Gonzalez, M. M., Dingus, B. L., Kaneko, Y., Preece, R. D., \& Briggs, M. S. 2004, in AIP Conf. Proc. 727: Gamma-Ray Bursts: 30 Years of Discovery, ed. E. E. Fenimore \& M. Galassi (Melville: AIP), 236

Götz, D., et al. 2006, A\&A, 445, 313

Guidorzi, C., Montanair, E., Frontera, F., Calura, F. Amati, L. Costa, E, \& Feroci, M. 2004, in ASP Conf. Ser. 312, Third Rome Workshop on GammaRay Bursts in the Afterglow Era, ed. M. Feroci et al. (San Francisco: ASP) 39

Helou, G., Khan, I. R., Malek, L., \& Boehmer, L. 1988, ApJS, 68, 151

Hurley, K., Briggs, M. S., Kippen, R. M., Kouveliotou, C., Meegan, C., Fishman, G., Cline, T., \& Boer, M. 1999, ApJS, 120, 399

Hurley, K. J., McBreen, B., Rabbette, M., \& Steel, S. 1994, A\&A, 288, L49

Hurley, K., et al. 2005, ApJS, 156, 217

Kippen, R. M., et al. 1998, ApJ, 492, 246

Kouveliotou, C., Meegan, C. A., Fishman, G. J., Bhat, N. P., Briggs, M. S.,

Koshut, T. M., Paciesas, W. S., \& Pendelton, G. M. 1993, ApJ 413, L101

Laros, J. G., et al. 1987, ApJ, 320, L111 1997, ApJS, 110, 157

Lazzati, D., Ghirlanda, G., \& Ghisellini, G. 2005, MNRAS, 362, L8 
Levan, A. J., Wynn, G. A., Chapman, R., Davies, M. B., King, A. R., Priddey, R. S., \& Tanvir, N. R. 2006, MNRAS, 368, L1

Mazets, E. P., Aptekar, R. L., Frederiks, D. D., Golenetskii, S. V., Il'Inskii, V. N., Palshin, V. D., Cline, T. L., \& Butterworth, P. S. 2004, in ASP Conf. Ser. 312, Third Rome Workshop on Gamma-Ray Bursts in the Afterglow Era, ed. M. Feroci et al. (San Francisco: ASP), 102

Nakar, E., Gal-Yam, A., Piran, T., \& Fox, D. B. 2006, ApJ, 640, 849

Ofek, E. O., et al. 2006, ApJ, 652, 507

Paczyński, B. 1992, Acta Astron., 42, 145

Palmer, D. M., et al. 2005, Nature, 434, 1107

Pietsch, W., et al. 2001, A\&A, 365, L174
Popov, S. B., \& Stern, B. E. 2006, MNRAS, 885

Sazonov, S. Y., Sunyaev, R. A., Terekhov, O. V., Lund, N., Brandt, S., \& Castro-Tirado, A. J. 1998, A\&AS, 129, 1

Scargle, J. D. 1982, ApJ, 263, 835

Stella, L., Dall'Osso, S., Israel, G. L., \& Vecchio, A. 2005, ApJ, 634, L165

Tkachenko, A. Y., Terekhov, O. V., Sunyaev, R. A., Kuznetsov, A. V., Barat, C., Dezalay, J.-P., \& Vedrenne, G. 2002, Astron. Lett., 28, 353

Tully, R. 1988, Nearby Galaxies Catalog (Cambridge: Cambridge Univ. Press) Vanderspek, R., Dullighan, A., \& HETE Science Team 2003, BAAS, 35, 623

Woods, P. M., \& Thompson, C. 2006, in Compact Stellar X-Ray Sources, ed. W. H. G. Lewin \& M. van der Klis (Cambridge: Cambridge Univ. Press), 547 